\title{
MiR-139-5p has an antidepressant-like effect by targeting phosphodiesterase 4D to activate the CAMP/PKA/CREB signaling pathway
}

\author{
Peng Huang ${ }^{1 \#} \wedge$, Songren Wei ${ }^{2 \#}$, Meng Luo ${ }^{3}$, Zhuohong Tang ${ }^{1}$, Qingmei Lin ${ }^{1}$, Xing Wang ${ }^{1}$, Mi Luo $^{1}$, \\ Yanjun $\mathrm{He}^{1}$, Chuan $\mathrm{Wang}^{4}$, Dezhan Wei ${ }^{1}$, Chenglai Xia ${ }^{1,5}$, Jiangping Xu ${ }^{2}$ \\ ${ }^{1}$ South Medical University Affiliated Maternal \& Child Health Hospital of Foshan, Foshan, China; ${ }^{2}$ Department of Neuropharmacology and Novel \\ Drug Discovery, School of Pharmaceutical Sciences, Southern Medical University, Guangzhou, China; ${ }^{3}$ Center for Bioinformatics, School of Life \\ Science and Technology, Harbin Institute of Technology, Harbin, China; ${ }^{4}$ Department of Biliary Surgery, The First People's Hospital of Foshan, \\ Foshan, China; ${ }^{5}$ School of Pharmaceutical Sciences, Southern Medical University, Guangzhou, China \\ Contributions: (I) Conception and design: J Xu, C Xia, P Huang; (II) Administrative support: P Huang; (III) Provision of study materials or patients: P \\ Huang, S Wei; (IV) Collection and assembly of data: M Luo, Y He, C Wang, D Wei; (V) Data analysis and interpretation: Z Tang, Q Lin, X Wang, \\ M Luo; (VI) Manuscript writing: All authors; (VII) Final approval of manuscript: All authors. \\ \#These authors contributed equally to this work. \\ Correspondence to: Chenglai Xia. South Medical University Affiliated Maternal \& Child Health Hospital of Foshan, Foshan, China. \\ Email: xiachenglai@126.com; Jiangping Xu. Department of Neuropharmacology and Novel Drug Discovery, School of Pharmaceutical Sciences, \\ Southern Medical University, Guangzhou, China. Email: jpx@smu.edu.cn.
}

Background: Phosphodiesterase 4D (PDE4D) inhibitor is commonly used to treat depression, but side effects seriously decrease its efficacy. PDE4D was a downstream target mRNA of miR-139-5p. Therefore, we examined the effects of hippocampal miR-139-5p gain- and loss-of-function on depression-like behaviors, the expression level of PDE4D, and hippocampus neurogenesis.

Methods: Bioinformatic analyses were carried out to to screen differential genes. Quantitative real-time polymerase chain reaction (qRT-PCR) and luciferase reporter assay were used to confirm the relationship between miR-139-5p and PDE4D. MiR-139-5p mimics, miR-139-5p inhibitor, or miR-NC were used to explore the function of miR-139-5p in HT-22 cells. We further explored the role of miR-139-5p in vivo using AAV-injection. Elisa, western blotting, and fluorescence in situ hybridization (FISH) were used to detect the expression of miR-139-5p and PDE4D in CRC tissues.

Results: Here, we showed that PDE4D messenger RNA (mRNA) was a direct target of microRNA (miR)-139-5p, which was downregulated in a chronic ultra-mild stress (CUMS)-induced depression mouse model. Moreover, in experiments in vitro, miR-139-5p mimic repressed PDE4D expression in HT-22 cells, but promoted phosphorylated cyclic-AMP response element-binding protein (p-CREB) and brain-derived neurotrophic factor (BDNF) expression. Interestingly, adeno-associated virus (AAV)miR-139-5p downregulated susceptibility to stress-induced depression-like behaviors in mice. AAV-miR139-5p suppressed PDE4D in mouse hippocampal cells, increasing expression level of cyclic adenosine monophosphate (cAMP), p-CREB, and BDNF, and stimulating mouse hippocampal neurogenesis.

Conclusions: Our findings suggested that miR-139-5p acted like an antidepressant by targeting PDE4D, thereby regulating the $\mathrm{cAMP} /$ protein kinase A (PKA)/CREB/BDNF pathway to improve depression.

Keywords: Depression; phosphodiesterase 4D (PDE4D); miR-139-5p; cyclic adenosine monophosphate (cAMP); brain-derived neurotrophic factor (BDNF)

$\wedge$ ORCID: 0000-0002-0187-0847. 
Submitted Aug 31, 2021. Accepted for publication Oct 22, 2021.

doi: $10.21037 /$ atm-21-5149

View this article at: https://dx.doi.org/10.21037/atm-21-5149

\section{Introduction}

Major depressive disorder (MDD), a common mental health disorder, will be the 2 nd leading cause of global disease burden by 2030 (1). However, a large number of MDD patients do not respond to medication currently available because of the poorly understood etiology of MDD $(2,3)$. Therefore, the development of effective antidepressants is urgently needed.

Phosphodiesterase 4 (PDE4), a cyclic adenosine monophosphate (cAMP)-specific phosphodiesterase (4), consists of 4 different isoforms: PDE4A, 4B, 4C, and 4D (5). A previous study reported that PDE4D was a key factor in inducing depression, mainly through the inhibition of the cAMP/protein kinase A (PKA)/cAMP-response element binding protein (CREB) signaling pathway $(6,7)$. However, PDE4D inhibitors, in turn, synthesize cAMP, which activates PKA, which phosphorylates CREB. Phosphorylated (p)-CREB translocates to the nucleus where it binds DNA elements and promotes brain-derived neurotrophic factor (BDNF) gene transcription, exerting an anti-depressive action through the enhancement of hippocampal neurogenesis (6). Unfortunately, systemic PDE4D inhibitors can cause emesis (8). Thus, the discovery of potent and selective PDE4D inhibitors could open new avenues for antidepressants.

Environmental challenges or stressful events, such as chronic ultra-mild stress (CUMS), alter microRNAs (miRNAs) in the brain, including the hippocampus $(9,10)$. MiRNAs are a class of small, noncoding RNAs able to mediate cleavage or translational repression of target mRNAs by binding preferentially to 3 ' untranslated regions (UTRs) (11). MiRNAs have been reported to regulate neurogenesis (12). Further, preclinical and clinical studies have demonstrated that miRNAs contribute to the pathophysiology of depression and to antidepressant drug actions (13).

To assess the potential contribution of hippocampal miRNAs to antidepressants, we measured the expression levels of several miRNAs directly targeting PDE4D in the hippocampus of mice exposed to CUMS. We found that miRNA-139-5p (miR-139-5p) was markedly suppressed, and PDE4D was a downstream target mRNA of miR-139-5p. MiR-139-5p/PDE4D mRNA interaction hinders PDE4D protein synthesis and initiates PDE4D mRNA degradation, which may be an effective way to reduce the side effects of drugs and therapeutic strategy for depression. Therefore, we examined the effects of hippocampal miR-139-5p gain- and loss-of-function on depression-like behaviors, the expression level of PDE4D, and hippocampus neurogenesis. We present the following article in accordance with the ARRIVE reporting checklist (available at https://dx.doi.org/10.21037/atm-21-5149).

\section{Methods}

\section{Animal care}

Eight-week-old C57BL/6, specific-pathogen free (SPF) male mice (weight $20-25 \mathrm{~g}$ ) were housed in a pathogenfree, temperature-controlled environment $\left(22 \pm 1^{\circ} \mathrm{C}\right)$, with a 12-hour light/12-hour dark cycle. Food and water were given ad libitum (apart from during model building). Animal experiments were performed under a project license (No. 2012-065) granted by ethics board of Southern Medical University, in compliance with Southern Medical University institutional guidelines for the care and use of animals.

\section{Experimental groups}

All animals were randomly assigned to various experimental groups ( $\mathrm{n}=10$ for each group): (I) control group (untreated group); (II) CUMS-exposed group, in which adult stress was imitated; (III) CUMS + adeno-associated virus (AAV)-miR139-5p group (injected with GFP mmu-miR-139-5p AAV miRNA virus); and (IV) CUMS+AAV-miR-139-5p sponge group (injected with GFP mmu-miR-139-5p sponge AAV miRNA virus).

\section{CUMS protocol and behavioral procedures}

CUMS modeling was performed with a previously described protocol $(14,15)$. Behavioral tests to evaluate depressivelike behavior in mice were performed during the light phase (9:00 AM to 3:00 PM), as described previously. 


\section{$A A V$-mediated gene transfer and injection}

We constructed the pAAV-CMA-bGI-mcs-EGFP-3GLAGmmu-miR-139-5p vector (AAV-miR- 139-5p, Figure S1A) and pAAV-CMA-bGI-mcs-EGFP-3GLAG-mmu-miR-139$5 \mathrm{p}$ sponge vector (AAV-miR-139-5p sponge, Figure $\mathrm{S} 1 \mathrm{~B}$ ) plasmid. Concentration of the above AAV vectors were both $6.0 \times 10^{12}$ viral genome $/ \mathrm{mL}$. Stereotaxic injection of AAV was performed as previously described $(16,17)$. AAVs were bilaterally infused into the mice using stereotaxic surgery (AP, $-2.0 \mathrm{~mm}$; ML, $\pm 1.5 \mathrm{~mm}$; DV,$-2.0 \mathrm{~mm}$ ) under sodium pentobarbital $(50 \mathrm{mg} / \mathrm{kg})$. Then, $1 \mu \mathrm{l}$ of AAV was infused into each hemisphere with a syringe pump at a flow rate of $0.05 \mu \mathrm{L} / \mathrm{min}$. After allowing 10 minutes for diffusion into the tissue, the injection needle was withdrawn, the skull was sealed with bone wax, and the wound was closed with surgical staples. Approximately 2 weeks after injection, brains were extracted and cut into $40 \mu \mathrm{m}$ frozen coronal sections. Green (excitation wavelength of $493 \mathrm{~nm}$ ) fluorescence of the injection area was observed with a fluorescence inversion microscope system. A fluorescent signal implied that the labeled gene was expressed and the injection placement was accurate.

\section{Cell cultures and drug intervention}

HT-22 cells (SCC129, Millipore, St Louis, MO, USA) were placed on 12-well plates and transfected using miR-139-5p mimics, miR-139-5p inhibitors, and miR-negative control (NC), according to the manufacturer's instructions, and were harvested 48 hours after transfection. The miR-139-5p mimics, miR-139-5p inhibitors, and miR-NC were purchased from Guangzhou RiboBio Co., Ltd. (Guangzhou, China).

\section{RNA extraction and miRNAseq analysis}

For RNA sequencing (RNAseq), RNA extraction from hippocampal tissues was performed using a Qiagen RNeasy mini kit in accordance with the manufacturer's instructions. Differential expression analysis was done using the edgeR Bioconductor software package, requiring q-values of $<0.05$ for both Wald and likelihood ratio tests and a beta value cut-off of 0.25 .

\section{Real-time polymerase chain reaction (PCR)}

Real-time PCR was performed as described previously (15). The primers for RT-PCR are listed in Table S1.

\section{Luciferase assay}

Luciferase reporter gene assay was used to detect the obligatory binding sites between miR-139-5p and PDE4D. Cotransfection with miR-NC and miR-159-5p mimics was done in 293T cells, which were collected from each well after 48 hours. The potency or activity of luciferase was detected through the GloMax Multi Detection System. Unpaired Student's t-test analysis was performed to measure the difference between groups.

\section{Western blot assay}

Western blotting was performed as described previously (15). The antibodies used are shown in Table S2.

\section{Mouse brain tissue immunofluorescence}

We previously reported detailed descriptions of the mouse brain tissue immunofluorescences $(16,17)$. Primary antibodies used are listed in Table S2.

\section{Fluorescent in situ bybridization (FISH)}

FISH was performed with a Fluorescent In Situ Hybridization Kit (RiboBio), following the manufacturer's protocols. Whole mouse brains were dissected and directly incubated overnight in fixing solution (phosphate-buffered saline (PBS) containing 1.6\% paraformaldehyde and 20\% sucrose), embedded in optimal cutting temperature (OCT) compound (Tissue-Tek, Sakura Finetek, Torrance, CA, USA), and snap frozen in 2-methylbutylene on dry-ice. The 40- $\mu \mathrm{m}$ sections were washed with ice-cold PBS (twice for 10 minutes), and blocking buffer [tris-buffered saline (TBS) containing $10 \%$ bovine serum albumin (BSA)] was added for 30 minutes. Sections were incubated with 0.45 $\mathrm{pmol} / \mu \mathrm{L}$ eubacterial oligonucleotide probe in prechilled hybridization buffer (Sigma-Aldrich, St Louis, MO, USA) overnight at $4{ }^{\circ} \mathrm{C}$. Sections were counterstained with $30 \mathrm{nM}$ 4',6-diamidino-2-phenylindole (DAPI) in PBS for 10 minutes, washed for 10 minutes in ice-cold PBS, and mounted with Fluoromount-G. Immunofluorescent imaging was performed on the same day.

\section{CAMP measurement assay}

CAMP levels in cell lysates were measured using a cAMP immunoassay kit according to the manufacturer's directions 
(Cayman Chemical, Ann Arbor, MI, USA).

\section{Statistical analyses}

Multiple group means were compared using an appropriate ANOVA test (1-way or 2-way). Significant effects were then evaluated with Tukey's post hoc tests or Bonferroni correction. Unpaired $t$-tests were used for 2 -group comparisons. In all cases, the tests were 2 -tailed and considered significant when $\mathrm{P} \leq 0.05$. All data are presented as mean \pm standard error of mean (SEM).

\section{Results}

MiR-139-5p was downregulated in the CUMS group compared to the control group, and PDE4D mRNA was a direct target of $\mathrm{miR}-139-5 \mathrm{p}$

In this study, we first identified the putative miRNAs (miR-139-5p, miR-335-5p, miR-340-5p, miR-7-5p, miR103a-3p, miR-19b-3p, miR-18a-5p, and miR-16-5p) targeting PDE4D using TargetScan and miRBase. Next, to validate the differential expression results, the above 8 predicted miRNAs were selected for qRT-PCR. Our data demonstrated that miR-139-5p, which had the most significant difference, was downregulated in the CUMS group compared to the control group (Figure 1A). We then explored the miRNA profile of mouse hippocampal tissues. MiRNA-seq analysis indicated that miR-139-5p exhibited the greatest downregulation (Figure 1B). Moreover, by searching the miR-139-5p seed sequence and mouse PDE4D 3'-UTR, we found that PDE4D 3'-UTR was complementary to the miR-139-5p sequence (Figure 1C). The results of the luciferase reporter assays confirmed this observation (Figure 1D). Finally, analysis of miR-139$5 \mathrm{p}$ and PDE4D mRNA expression by fluorescent in situ hybridization revealed that miR-139-5p was located in the nucleus of mouse hippocampal cells, while PDE4D mRNA existed in the cytoplasm (Figure 1E).

\section{MiR-139-5p repressed PDE4D expression in HT-22 cells, promoted p-CREB and BDNF expression, and resulted in no change in CREB}

To investigate whether miR-139-5 p could up- or downregulate PDE4D, we assessed PDE4D mRNA and protein expression in miR-139-5p mimic, miR-139$5 \mathrm{p}$ inhibitors, or miR-NC-transfected HT-22 cells. We found that miR-139-5p mimics elevated miR-139-5p and BDNF mRNA levels but inhibited PDE4D mRNA levels (Figure 2A). However, these expression levels were reversed by miR-139-5p inhibitor. In addition, miR-139-5p repressed PDE4D protein expression (Figure 2B,2C), promoted p-CREB (Figure 2B,2D) and BDNF (Figure 2B,2E) protein expression, while there was no change in CREB (Figure $2 F$ ) protein levels. Similarly, miR-139-5p inhibitor reversed the above protein expression levels.

\section{AAV-miR-139-5p downregulated susceptibility to stress- induced depression-like behaviors in mice}

The experimental setup is shown in Figure $3 A$. After subjected to CUMS for 6 weeks, mice were injected with AAV-miR-139-5p or AAV-miR-139-5p sponge into the bilateral hippocampus. A schematic diagram of AAV-injection is shown in Figure $3 B$, and the transgene expression (GFP, green) after vector microinjection is shown in Figure 3C. As is apparent, AAV-miR-139-5p upregulated miR-139-5p in AAV-GFP-injected mice (Figure 3D). Next, depression-like behavior was assessed using the sucrose preference test (SPT), forced swimming test (FST), and tail suspension test (TST). Interestingly, AAV-miR-139-5p significantly increased SPT scores (Figure $3 E$ ) and attenuated the increased immobility time of FST (Figure 3F) and TST (Figure 3G) in mice. Conversely, AAV-miR-139-5p sponge injections aggravated depressivelike behaviors (Figure 3E-3G).

\section{MiR-139-5p suppressed PDE4D in mouse hippocampal cells, increasing expression level of cAMP, $p$-CREB, and $B D N F$, and stimulating mice hippocampal neurogenesis}

Our results showed that CUMS-exposed mice had elevated PDE4D protein levels, which were reversed by miR-139-5p (Figure $4 A, 4 B$ ). In parallel, we found that CUMS-exposed mice showed low expression of cAMP (Figure $4 C$ ), p-CREB (Figure $4 A, 4 D$ ), and BDNF (Figure $4 A, 4 E$ ) protein. These findings were reversed in the AAV-miR-139-5p group, with miR-139-5p upgrading cAMP levels (Figure 4C) and also p-CREB and BDNF protein expression levels (Figure $4 A, 4 D, 4 E)$. However, the opposite occurred for AAVmiR-139-5p sponge (Figure 4A-E). There was no change in CREB (Figure $4 A, 4 F$ ) protein levels. To understand the effects of miR-139-5p on neurogenesis in the hippocampus, we investigated the population of bromodeoxyuridine positive $\left(\mathrm{BrdU}^{+}\right)$, doublecortin positive $\left(\mathrm{DCX}^{+}\right)$, and Nestin 
A

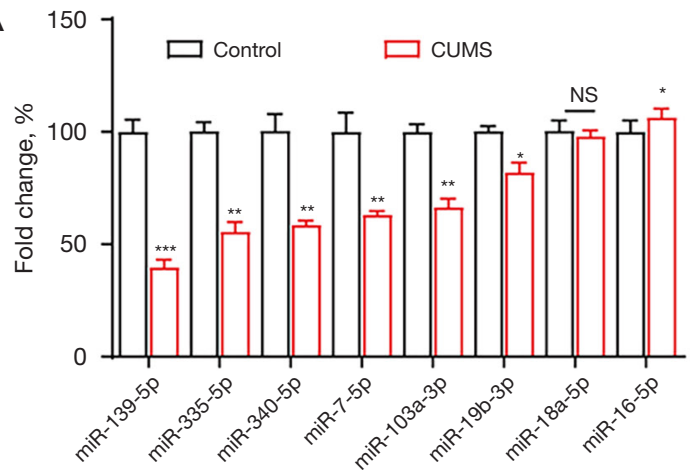

C

\section{Position 4308-4315 of PDE4D 3' UTR}

WT PDE4D 3' UTR 5 ' ...UAUUUUUUGACACAA--ACUGUAGA...-3' hsa-miR-139-5p 3' UGACCUCUGUGCACGUGACAUCU-5' Mut PDE4D 3' UTR 5 ' ...UAUUUUUUGACACAA--CAGCCGA...-3'

B

$\mathrm{D}$
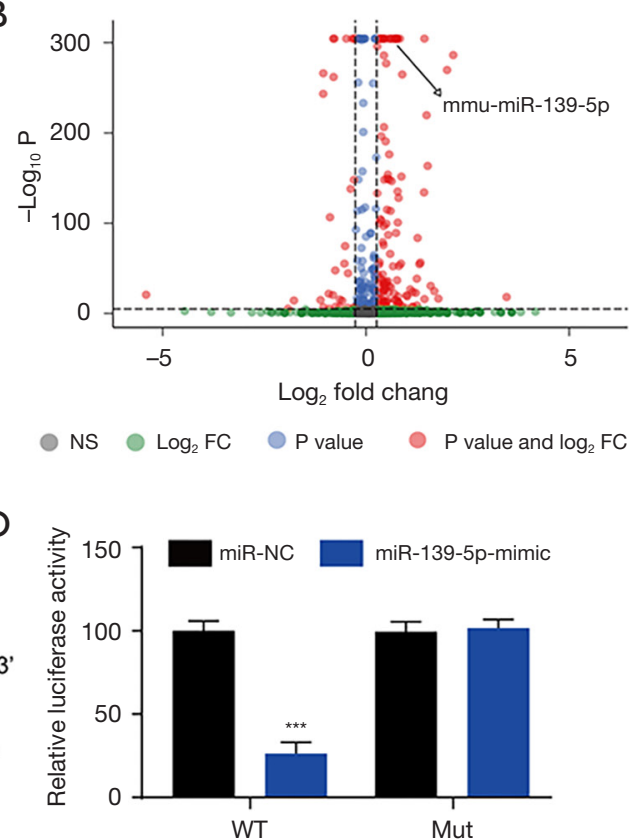

PDE4D

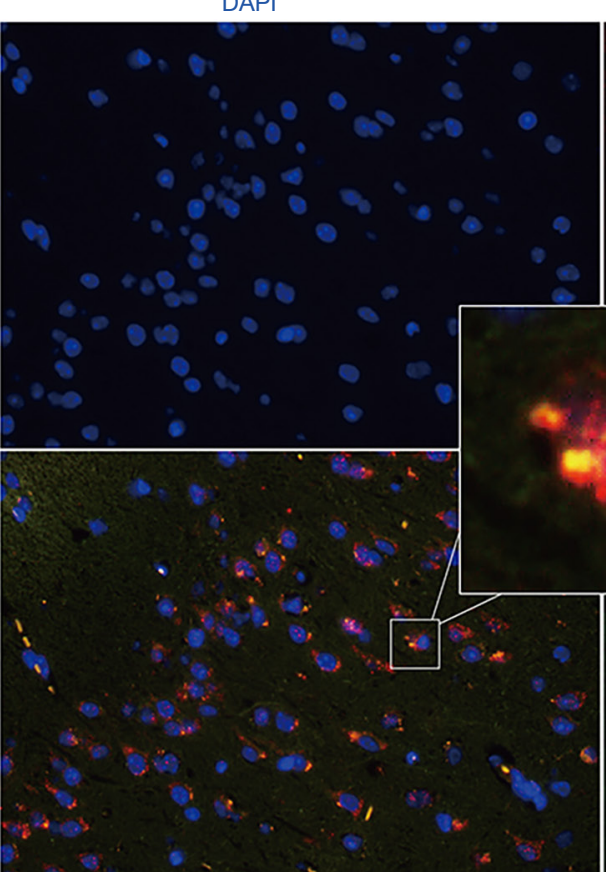

Megger

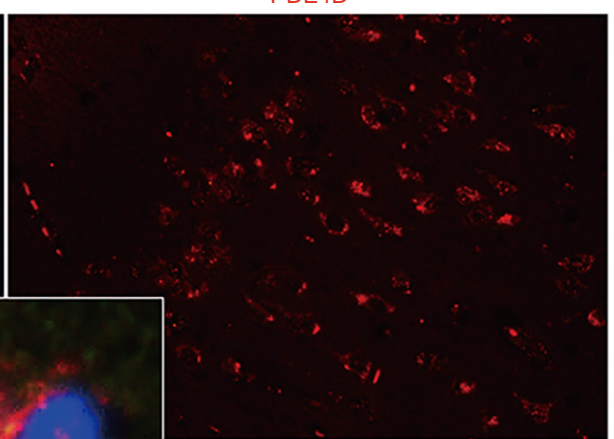

Figure 1 MiR-139-5p was downregulated in the CUMS group compared to the control group, and PDE4D was a direct target of miR-139$5 \mathrm{p}$ in mouse hippocampal cells. (A) MiR-139-5p was downregulated in the CUMS group compared to the control group; (B) MiRNAseq analysis indicated that miR-139-5p exhibited the greatest downregulation; (C) alignment of potential binding sites for miR-139-5p in the 3' UTRs of PDE4D mRNA; (D) luciferase reporter assays showed that miR-139-5p targeted PDE4D 3' UTR; (E) fluorescent in situ hybridization analysis. MiR-139-5p and PDE4D mRNAs in a cell were visualized using single molecule FISH probes. Nuclei were counterstained with Hoechst 33342. Scale bar $=50 \mu \mathrm{m}$. ${ }^{*} \mathrm{P} \leq 0.05,{ }^{* *} \mathrm{P} \leq 0.01$, and ${ }^{* * *} \mathrm{P} \leq 0.01$ versus the control group. All data are presented as mean \pm SEM. CUMS, chronic ultra-mild stress; PDE4D, phosphodiesterase 4D; 3' UTR, 3' untranslated region. 
A

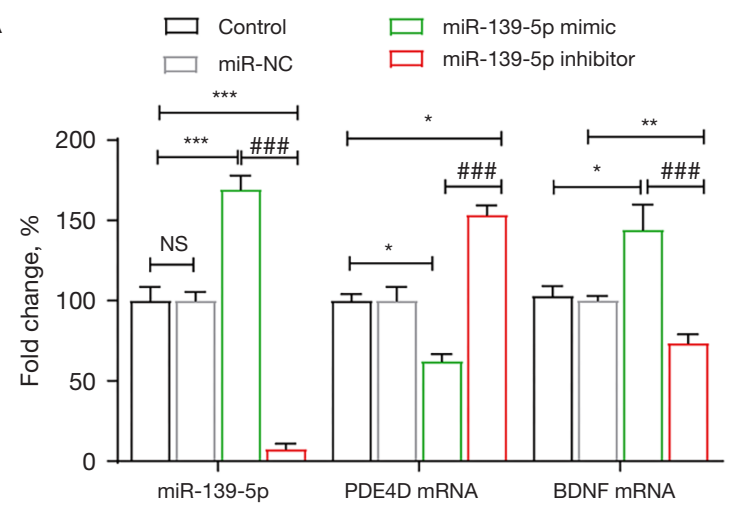

C

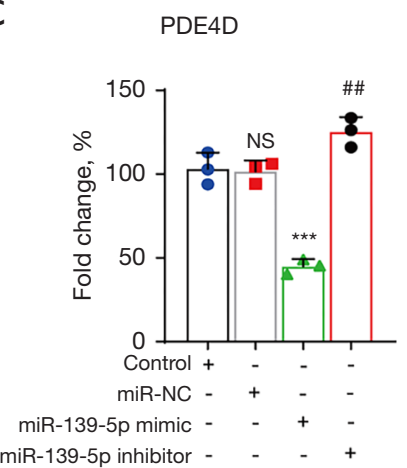

B

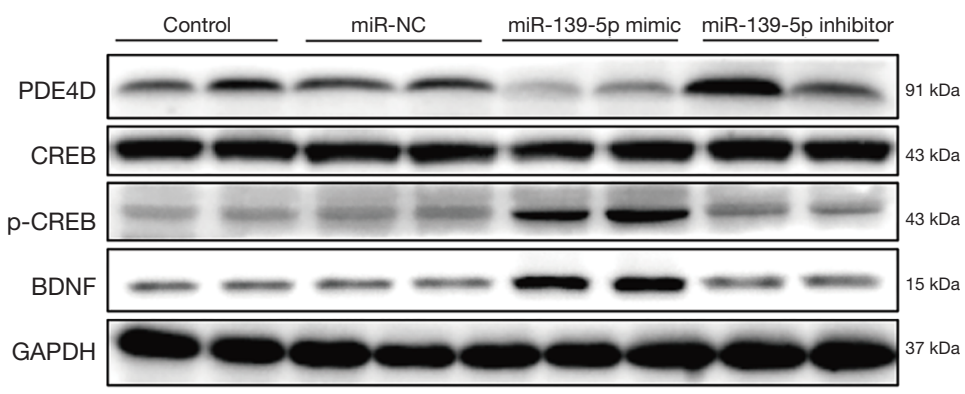

$E$

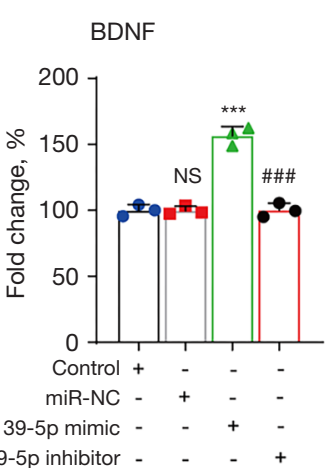

$\mathrm{F}$

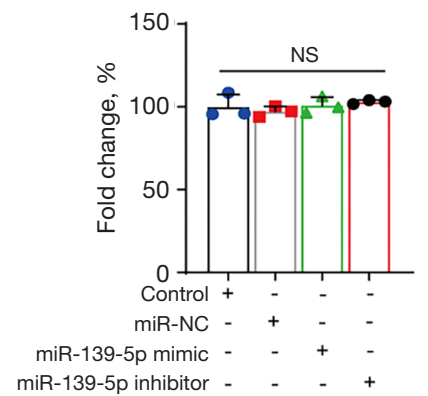

Figure 2 MiR-139-5p repressed PDE4D expression in HT-22 cells but promoted p-CREB and BDNF expression, while there was no change in CREB. (A) Levels of mRNAs for miR-139-5p, PDE4D, and BDNF mRNA in HT-22 cells transfected with miR-139-5p mimics, miR-139-5p inhibitor, or miR-NC. MiR-139-5p mimics showed elevated miR-139-5p and BDNF mRNA levels but inhibited PDE4D mRNA levels. These expression levels were reversed by miR-139-5p inhibitor. (B-F) Protein levels of PDE4D, p-CREB, BDNF, and CREB in HT-22 cells transfected with miR-139-5p mimics, miR-139-5p inhibitor, or miR-NC. MiR-139-5p repressed PDE4D (C) protein expression but promoted p-CREB (D) and BDNF (E) protein expression, while there was no change in CREB (F) protein levels. $\mathrm{n}=10$ mice per group. ${ }^{*} \mathrm{P} \leq 0.05,{ }^{* *} \mathrm{P} \leq 0.01$, and ${ }^{* * *} \mathrm{P} \leq 0.01$ versus the control group; ${ }^{\# \#} \mathrm{P} \leq 0.01$ and ${ }^{\# \# \#} \mathrm{P} \leq 0.001$ versus miR-139-5p mimic group. All data are presented as mean \pm SEM. PDE4D, phosphodiesterase 4D; p-CREB, phosphorylated cyclic-AMP response element-binding protein; BDNF, brain-derived neurotrophic factor.

positive cells in hippocampal regions. Unsurprisingly, compared with the control group, $\mathrm{BrdU}^{+}$(Figure 4G,4H), $\mathrm{DCX}^{+}$(Figure 4G,4I), and Nestin ${ }^{+}$(Figure 4G,4f) cells in mouse hippocampus were significantly reduced in the CUMS group. However, with the application of AAVmiR-139-5p, miR-139-5p significantly increased these cell populations, while the exact opposite was observed in the AAV-miR-139-5p sponge group (Figure 4G-4I).

\section{Discussion}

The PDE4 gene family consists of four subtypes (PDE4A-D) and more than 20 splice variants (18). PDE4D is located in chromosome $5 \mathrm{q} 12$ and encodes cAMP-specific 3',5'-cyclic phosphodiesterase 4D (19). PDE4D mRNA is mainly expressed in the emetic center of the mouse hippocampus (such as the nucleus tractus solitarius), but it is upregulated after CUMS mode (20). Studies now imply that selective inhibition of the PDE4D subtype may conserve antidepressant effect and decrease sideeffect liability, whereas genetic linkage studies potentially associate PDE4B gene variants with schizophrenia (18). Similar to other studies, we found that PDE4D expression was increased in CUMS-induced depression mice (Figure S1C,S1D). Accordingly, we proposed targeting PDE4D as a potential therapeutic approach. Recently, the efficacy of PDE4D inhibitors has been investigated. Among the first generation of well-known and clinically tested 


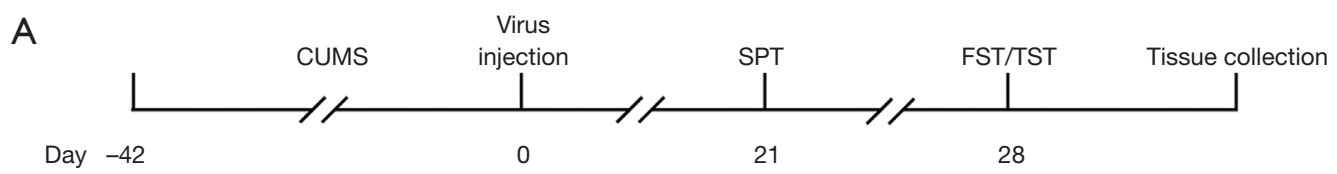

B

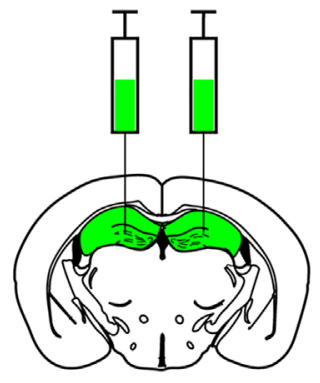

Hippocampus
C

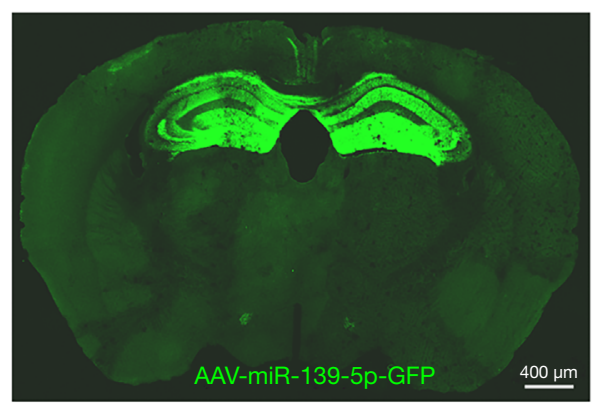

D

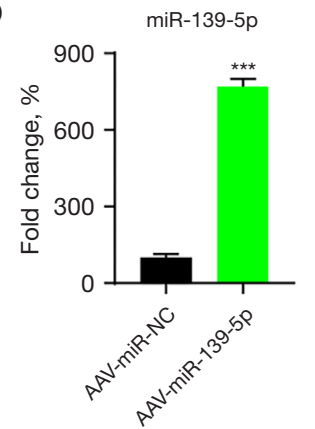

E

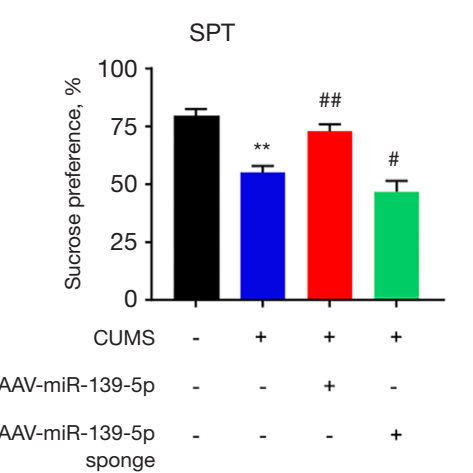

$\mathrm{F}$

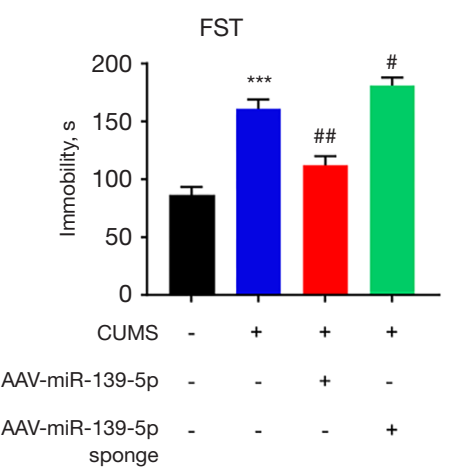

G

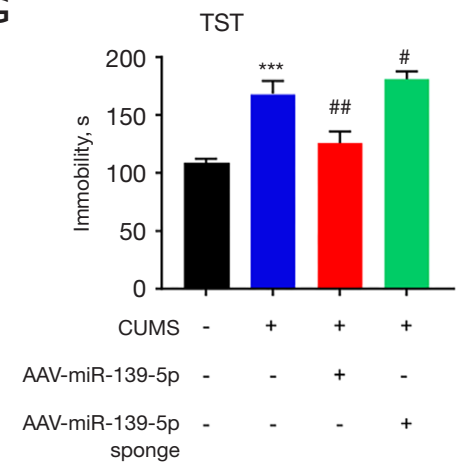

Figure 3 AAV-miR-139-5p downregulated susceptibility to stress-induced depression-like behaviors in mice. (A) Schematic diagram of the experimental design. After subjected to CUMS for 6 weeks, mice were injected with AAV-miR-139-5p or AAV-miR-139-5p sponge into the bilateral hippocampus. Mice underwent SPT, FST, and TST in behavioral assays. All mice were then killed for expression analysis. (B) Schematic diagram of AAV-injection. Transgene expression (GFP, green) after vector microinjection is shown in (C). (D) AAV-miR-1395p upregulated miR-139-5p in AAV-GFP-injected mice. (E-G) Effects of AAV-miR-39-5p overexpression or inhibitor on depression-like behaviors. Briefly, AAV-miR-139-5p increased sucrose preference (E) and reduced immobility time (F-G). n=10 mice per group. Scale bar $=400 \mu \mathrm{m} .{ }^{* *} \mathrm{P} \leq 0.01$ and ${ }^{* * *} \mathrm{P} \leq 0.01$ versus the control group; ${ }^{*} \mathrm{P} \leq 0.05$ and ${ }^{\# \#} \mathrm{P} \leq 0.01$ versus the CUMS group. All data are presented as mean \pm SEM. AAV, adeno-associated virus; CUMS, chronic ultra-mild stress.

PDE4 inhibitors, the prototype rolipram was the first selective PDE4 inhibitor with potent anti-inflammatory actions to be studied (21). However, severe unacceptable adverse events, including nausea and vomiting, blocked the clinical application of rolipram (22). Researchers subsequently invented apremilast, a newer third-generation PDE4 inhibitor, by using a recognized functional pharmacophore of earlier PDE4 inhibitors and adding a series of active groups in order to reduce side effects and optimize pharmaceutical effects (23). However, clinical evidence suggests that treatment with repeated dosing of roflumilast may lead to the development of tachyphylaxis or tolerance for roflumilast through up-regulation of PDE4B expression (24). Nevertheless, to date, there have been no studies reporting an effective new PDE4D inhibitor without side effects. Therefore, it is necessary to search for new medications that target PDE4D.

MicroRNAs are small non-coding RNAs ( 22 nt) which are involved in key biological processes such as cell proliferation, cell apoptosis, and metabolism (25). 


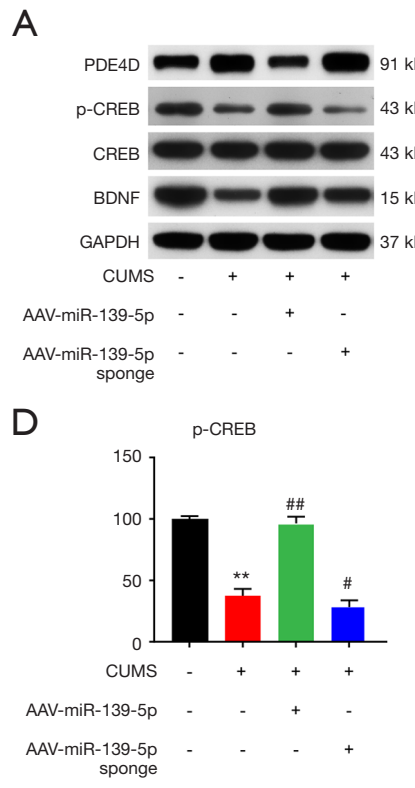

\section{B}

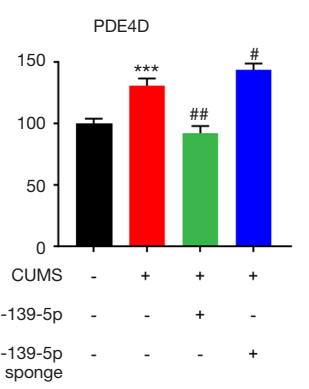

C
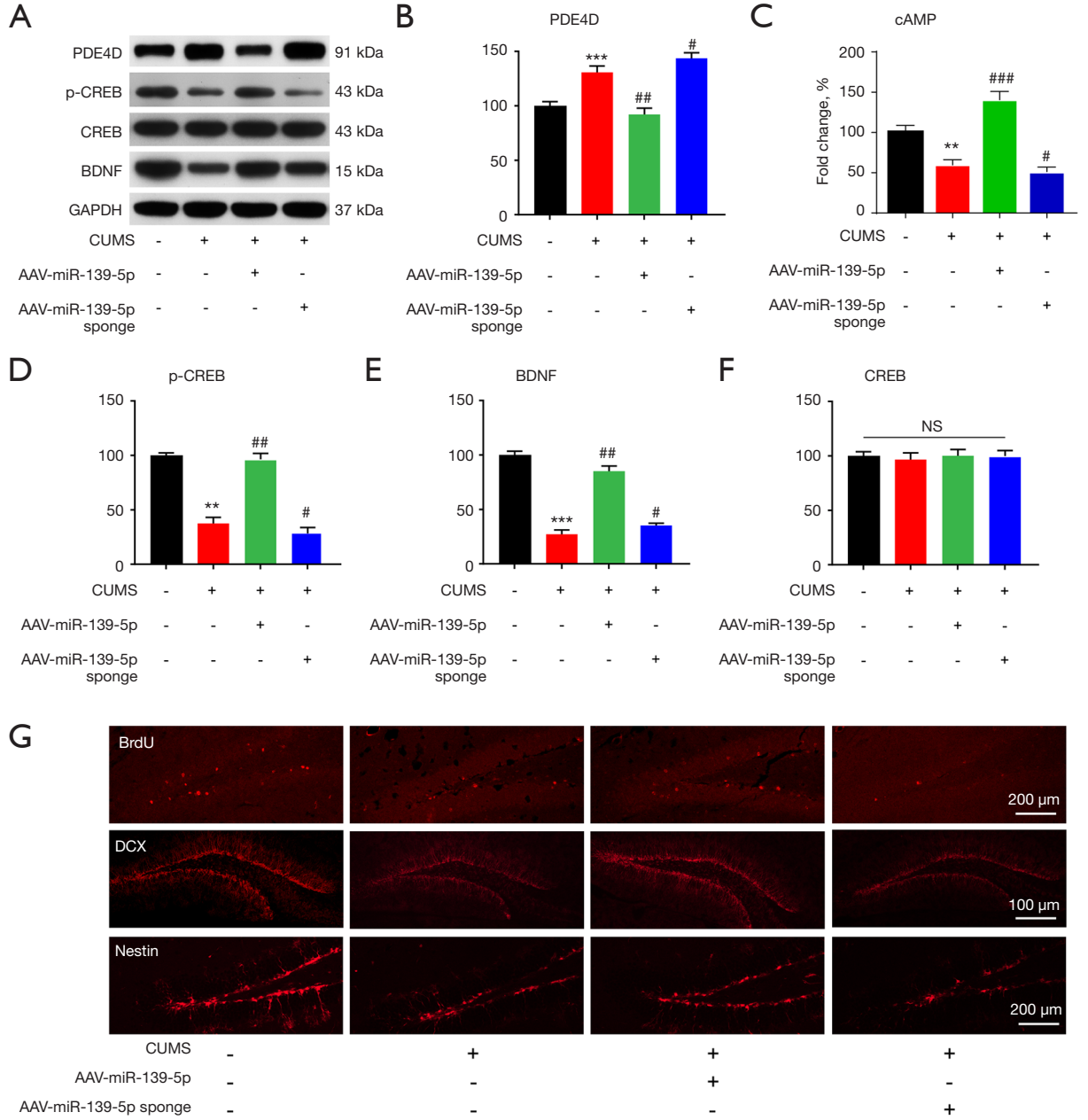

AAV-miR-139-5p sponge
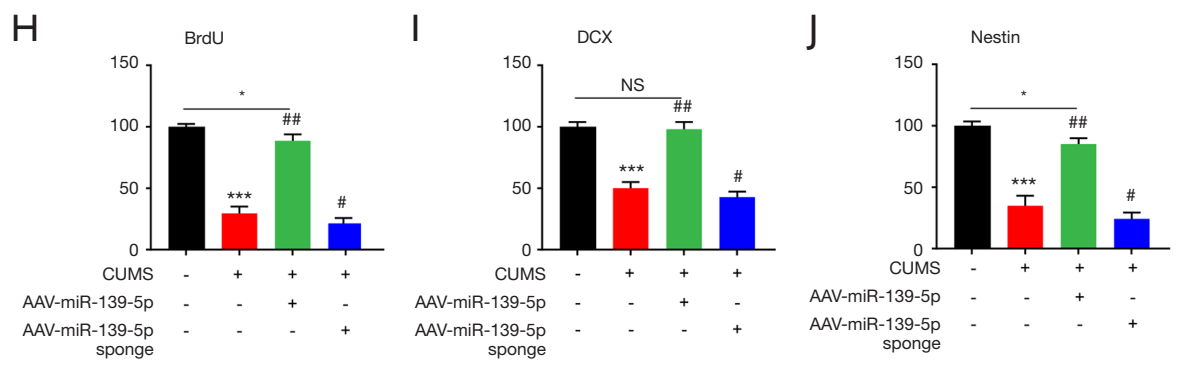

Figure 4 MiR-139-5p suppressed PDE4D in mouse hippocampal cells, increasing expression levels of cAMP, p-CREB, and BDNF, and stimulating mouse hippocampal neurogenesis. (A) Western blot. (B) CUMS-exposed mice showed elevated PDE4D protein level, which was reversed by miR-139-5p. (C) Elisa: levels of cAMP in the hippocampus of mice subjected to a 6-week CUMS session and AAV-injection. CUMS-exposed mice showed low expression cAMP levels, but this was reversed by miR-139-5p. (D-F) Protein levels of p-CREB, BDNF, and CREB in the hippocampus of mice subjected to 6-week CUMS. MiR-139-5p also upgraded p-CREB (D) and BDNF (E) protein expressions in AAV-miR-139-5p group mice compared with CUMS-exposed mice. There was no change in CREB protein (F) levels. (GJ) Effects of miR-139-5p on neurogenesis in the hippocampus. Red fluorescence indicated BrdU (G-H), DCX (G,I), and Nestin (G,J), and then quantified the immunofluorescence signals. $\mathrm{n}=10$ mice per group. Scale bar $=200 \mu \mathrm{m} .{ }^{* *} \mathrm{P} \leq 0.01$ and ${ }^{* * *} \mathrm{P} \leq 0.01$ versus the control group; ${ }^{\#} \mathrm{P} \leq 0.05,{ }^{\# \#} \mathrm{P} \leq 0.01$, and ${ }^{\# \# \#} \mathrm{P} \leq 0.001$ versus CUMS group. All data are presented as mean \pm SEM. PDE4D, phosphodiesterase 4D; p-CREB, phosphorylated cyclic-AMP response element-binding protein; BDNF, brain-derived neurotrophic factor; CUMS, chronic ultra-mild stress; $\mathrm{AAV}$, adeno-associated virus; BrdU, bromodeoxyuridine; DCX, doublecortin. 
MicroRNAs achieve their biological function by binding to mature mRNAs and in most cases, decreasing the expression level of target mRNAs (26). The association between microRNA and human disease can be converted into an association between microRNA-mRNA interaction and human disease, thereby providing a means to influence microRNA-regulated human diseases (27). There is accumulating evidence indicating a significant miRNA contribution to depression, anxiety, and antidepressant action $(28,29)$. A recent preclinical study showed that upregulated miRNA in the sperm of patients with depression significantly inhibited the expression of key genes in the pathway (30). In another study, miRNA-26a-3p was reported to be a putative modulator of antidepressant response (31). Therefore, posttranscriptional regulation by miRNA networks in various brain regions is likely involved in depression and antidepressant drug actions.

We demonstrated that miR-139-5p targeted PDE4D (Figure 1). First, 8 candidate miRNAs were screened. Among them, miR-139-5p, which had the most significant difference, was downregulated in the CUMS group compared to the control group. Next, the PDE4D mRNA sequence targeted by miR-139-5p was predicted using TargetScan and miRBase. We then assessed luciferase expression driven by either a wild type or mutant PDE4Dmotif-containing miR-139 sequence in $293 \mathrm{~T}$ cells. Finally, our findings were further confirmed by double color in situ hybridization. The above results clearly suggested that PDE4D mRNA is a direct target of miR-139-5p.

We also found that hippocampal miR-139-5p mediated behavioral responses to chronic stress in mice. Increasing hippocampal miR-139-5p blocked depression-like behaviors caused by chronic stress, resulting in a state of stress resilience. Conversely, inhibition of hippocampal miR-139$5 \mathrm{p}$ enhanced behavioral susceptibility to a CUMS protocol (Figure 3).

Remarkably, we found that by targeting PDE4D, miR139-5p powerfully regulated the cAMP/PKA/CREB/BDNF pathway to facilitate hippocampal neurogenesis (Figure 2). We showed in an in vitro assay that the expression of PDE4D was regulated by miR-139-5p. MiR-139-5p repressed PDE4D expression in HT-22 cells but promoted p-CREB and BDNF expression, while there was no change in CREB. In addition, AAV-miR-139-5p and sponge viruses were injected into the hippocampus of CUMS mice. It is evident in Figure 3C,3D that the miR-139-5p expression was significantly enhanced after AAV-miR-139$5 \mathrm{p}$ injection. Our results showed that CUMS-exposed mice showed elevated PDE4D protein levels and low expression of cAMP, p-CREB, and BDNF proteins. However, AAVmiR-139-5p inhibited the expression of PDE4D protein and also upgraded cAMP, p-CREB, and BDNF protein expression. To further investigate the mechanism of miR$139-5 p$ on depression, we sought to determine whether the antidepressant effects of miR-139-5p were associated with hippocampal neurogenesis, mediated by the PDE4DcAMP/PKA/CREB/BDNF pathway. We found that, compared with the CUMS group, $\mathrm{BrdU}^{+}, \mathrm{DCX}^{+}$, and $\mathrm{Nestin}^{+}$cells in mouse hippocampus were significantly increased in the AAV-miR-139-5p group. Therefore, AAVmiR-139-5p treatment ameliorated the CUMS-induced impairment of hippocampal neurogenesis. However, the exact opposite was observed in the AAV-miR-139-5p sponge group. Together, these results showed that miR-139-5p exerted an antidepressant effect and regulated the cAMP/ PKA/CREB/BDNF pathway by targeting PDE4D.

A previous study by Wei et al. (32) reported that miR139-5p from exosomes of patients with major depression caused depression-like behavior in mice, which is in direct contradiction to our findings. However, Cao et al. (33) found a significant inverse correlation between the expression of miR-139-5p and PDE4D, with overexpression of miR-139-5p decreasing PDE4D levels and increasing cellular cAMP levels, supporting our theory that miR$139-5 p$ suppressed levels of PDE4D. Differences in model species, sample source, genetic background, and their interactions may account for the discrepancies between our results and those of Wei et al. We used hippocampus tissue of C57BL/6 mice subjected to CUMS, whereas Wei et al. used blood-derived exosomes from MDD patients. It is generally known that the contents of bioactive compounds (including gene and miRNA expression) are significantly different in samples from different sources (34). Therefore, sample source and genetic background may have unique effects on gene expression profiles. Another possible explanation for this discrepancy is the treatment approach. We used AAV vectors to overexpress miR-139$5 \mathrm{p}$ treatment specifically in hippocampus neurons, whereas Wei et al. used injection of blood exosomes isolated from MDD patients and treated mice were rescued by intranasal injection of miR-139-5p antagomir. Therefore, miR-139-5p overexpression in different species and tissues may produce disparate behavioral outcomes. Nonetheless, our results emphasize the importance of miRNA dysregulation in the pathogenesis of depression.

In conclusion, we demonstrated that hippocampal 
miR-139-5p directly targeted PDE4D. Furthermore, an upregulation of miR-139-5p in adult mouse hippocampus ameliorated depression-like behavior and increased hippocampal neurogenesis. Together, these results showed that miR-139-5p regulated the cAMP/PKA/ CREB/BDNF pathway by targeting PDE4D to improve depression. Another important implication of this study was that miR-139-5p pathways may contribute to the gene regulation required for antidepressant drug action, such as PDE4D inhibitors. Compared with rolipram and apremilast, miR-139-5p/PDE4D mRNA interaction hinders PDE4D protein synthesis and initiates PDE4D mRNA degradation, which may an effective way to reduce the side effects of drugs. Therefore, direct modulation of miRNA pathways may be an effective therapeutic strategy for depression.

\section{Acknowledgments}

Funding: This work was supported by the National Natural Science Foundation of China (81901362), the China Postdoctoral Science Foundation (2019M652969), Traditional Chinese Medicine Bureau of Guangdong Province Scientific Research Project (20201340), and the Bureau of Science and Technology of Foshan City Project (1920001000269 and 1920001000475).

\section{Footnote}

Reporting Checklist: The authors have completed the ARRIVE reporting checklist. Available at https://dx.doi. org/10.21037/atm-21-5149

Data Sharing Statement: Available at https://dx.doi. org/10.21037/atm-21-5149

Conflicts of Interest: All authors have completed the ICMJE uniform disclosure form (available at https://dx.doi. org/10.21037/atm-21-5149). The authors have no conflicts of interest to declare.

Ethical Statement: The authors are accountable for all aspects of the work in ensuring that questions related to the accuracy or integrity of any part of the work are appropriately investigated and resolved. Animal experiments were performed under a project license (No. 2012-065) granted by ethics board of Southern Medical University, in compliance with Southern Medical University institutional guidelines for the care and use of animals.

Open Access Statement: This is an Open Access article distributed in accordance with the Creative Commons Attribution-NonCommercial-NoDerivs 4.0 International License (CC BY-NC-ND 4.0), which permits the noncommercial replication and distribution of the article with the strict proviso that no changes or edits are made and the original work is properly cited (including links to both the formal publication through the relevant DOI and the license). See: https://creativecommons.org/licenses/by-nc$\mathrm{nd} / 4.0 /$.

\section{References}

1. Geugies H, Mocking RJT, Figueroa CA, et al. Impaired reward-related learning signals in remitted unmedicated patients with recurrent depression. Brain 2019;142:2510-22.

2. Fogaça MV, Fukumoto K, Franklin T, et al. N-Methyl$\mathrm{D}$-aspartate receptor antagonist $\mathrm{d}$-methadone produces rapid, mTORC1-dependent antidepressant effects. Neuropsychopharmacology 2019;44:2230-8.

3. Smalheiser NR, Zhang H, Dwivedi Y. Enoxacin Elevates MicroRNA Levels in Rat Frontal Cortex and Prevents Learned Helplessness. Front Psychiatry 2014;5:6.

4. Bolger GB, Smoot LHM, van Groen T. DominantNegative Attenuation of cAMP-Selective Phosphodiesterase PDE4D Action Affects Learning and Behavior. Int J Mol Sci 2020;21:5704.

5. Beute J, Lukkes M, Koekoek EP, et al. A pathophysiological role of PDE3 in allergic airway inflammation. JCI Insight 2018.

6. Zou ZQ, Chen JJ, Feng HF, et al. Novel Phosphodiesterase 4 Inhibitor FCPR03 Alleviates Lipopolysaccharide-Induced Neuroinflammation by Regulation of the cAMP/PKA/CREB Signaling

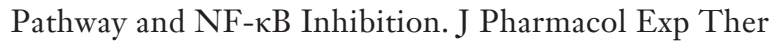
2017;362:67-77.

7. Xu B, Wang T, Xiao J, et al. FCPR03, a novel phosphodiesterase 4 inhibitor, alleviates cerebral ischemia/reperfusion injury through activation of the AKT/GSK3 $\beta / \beta$-catenin signaling pathway. Biochem Pharmacol 2019;163:234-49.

8. Cavalloro V, Russo K, Vasile F, et al. Insight into GEBR-32a: Chiral Resolution, Absolute Configuration and Enantiopreference in PDE4D Inhibition. Molecules 2020;25:935. 
9. Xiao Y, Luo H, Yang WZ, et al. A Brain Signaling Framework for Stress-Induced Depression and Ketamine Treatment Elucidated by Phosphoproteomics. Front Cell Neurosci 2020;14:48.

10. Katoh M. Cardio-miRNAs and onco-miRNAs: circulating miRNA-based diagnostics for non-cancerous and cancerous diseases. Front Cell Dev Biol 2014;2:61.

11. Son YO, Kim HE, Choi WS, et al. RNA-binding protein ZFP36L1 regulates osteoarthritis by modulating members of the heat shock protein 70 family. Nat Commun 2019;10:77.

12. Mukherjee S, Akbar I, Bhagat R, et al. Identification and Classification of Hubs in microRNA Target Gene Networks in Human Neural Stem/Progenitor Cells following Japanese Encephalitis Virus Infection. mSphere 2019;4:00588-19.

13. Zhang HP, Liu XL, Chen JJ, et al. Circulating microRNA 134 sheds light on the diagnosis of major depressive disorder. Transl Psychiatry 2020;10:95.

14. Huang P, Gao T, Dong Z, et al. Neural circuitry among connecting the hippocampus, prefrontal cortex and basolateral amygdala in a mouse depression model: Associations correlations between BDNF levels and BOLD - fMRI signals. Brain Res Bull 2018;142:107-15.

15. Gao L, Huang P, Dong Z, et al. Modified Xiaoyaosan (MXYS) Exerts Anti-depressive Effects by Rectifying the Brain Blood Oxygen Level-Dependent fMRI Signals and Improving Hippocampal Neurogenesis in Mice. Front Pharmacol 2018;9:1098.

16. Gao L, Gao T, Zeng T, et al. Blockade of Indoleamine 2, 3-dioxygenase 1 ameliorates hippocampal neurogenesis and BOLD-fMRI signals in chronic stress precipitated depression. Aging (Albany NY) 2021;13:5875-91.

17. Wang Z, Li J, Wu W, et al. Saikosaponin D Rescues Deficits in Sexual Behavior and Ameliorates Neurological Dysfunction in Mice Exposed to Chronic Mild Stress. Front Pharmacol 2021;12:625074.

18. Wakabayashi Y, Telu S, Dick RM, et al. Discovery, Radiolabeling, and Evaluation of Subtype-Selective Inhibitors for Positron Emission Tomography Imaging of Brain Phosphodiesterase-4D. ACS Chem Neurosci 2020;11:1311-23.

19. Song YL, Wang CJ, Wu YP, et al. Phosphodiesterase 4D polymorphisms associate with the short-term outcome in ischemic stroke. Sci Rep 2017;7:42914.

20. Zhang C, Cheng Y, Wang H, et al. RNA interferencemediated knockdown of long-form phosphodiesterase-4D (PDE4D) enzyme reverses amyloid- 42-induced memory deficits in mice. J Alzheimers Dis 2014;38:269-80.

21. Saha A, Bhattacharjee A, Vij A, et al. Evaluation of Modulators of cAMP-Response in Terms of Their Impact on Cell Cycle and Mitochondrial Activity of Leishmania donovani. Front Pharmacol 2020;11:782.

22. Kumar N, Goldminz AM, Kim N, et al.

Phosphodiesterase 4-targeted treatments for autoimmune diseases. BMC Med 2013;11:96.

23. Chen Y, Li Z, Li H, et al. Apremilast Regulates the Teff/Treg Balance to Ameliorate Uveitis via PI3K/ AKT/FoxO1 Signaling Pathway. Front Immunol 2020;11:581673.

24. Lee BC, Susuki-Miyata S, Yan C, et al. Dexamethasone Inhibits Synergistic Induction of PDE4B Expression by Roflumilast and Bacterium NTHi. Int J Mol Sci 2018;19:3511.

25. Sim DY, Lee HJ, Jung JH, et al. Suppression of STAT3 Phosphorylation and RelA/p65 Acetylation Mediated by MicroRNA134 Plays a Pivotal Role in the Apoptotic Effect of Lambertianic Acid. Int J Mol Sci 2019;20:2993.

26. Fernandes J, Miranda RL, de Lemos ERS, et al. MicroRNAs and Mammarenaviruses: Modulating Cellular Metabolism. Cells 2020;9:2525.

27. Wu D, Yang G, Zhang L, et al. Genome-wide association study combined with biological context can reveal more disease-related SNPs altering microRNA target seed sites. BMC Genomics 2014;15:669.

28. Miao N, Jin J, Kim SN, et al. Hippocampal MicroRNAs Respond to Administration of Antidepressant Fluoxetine in Adult Mice. Int J Mol Sci 2018;19:671.

29. Casselli T, Qureshi H, Peterson E, et al. MicroRNA and mRNA Transcriptome Profiling in Primary Human Astrocytes Infected with Borrelia burgdorferi. PLoS One 2017;12:e0170961.

30. Wang Y, Chen ZP, Hu H, et al. Sperm microRNAs confer depression susceptibility to offspring. Sci Adv 2021;7:eabd7605.

31. Li Y, Fan C, Wang L, et al. MicroRNA-26a-3p rescues depression-like behaviors in male rats via preventing hippocampal neuronal anomalies. J Clin Invest 2021;131:148853.

32. Wei ZX, Xie GJ, Mao X, et al. Exosomes from patients with major depression cause depressive-like behaviors in mice with involvement of miR-139-5pregulated neurogenesis. Neuropsychopharmacology 2020;45:1050-8.

33. Cao B, Wang K, Liao JM, et al. Inactivation of oncogenic cAMP-specific phosphodiesterase 4D 
Page 12 of 12

by $\mathrm{miR}-139-5 \mathrm{p}$ in response to $\mathrm{p} 53$ activation. Elife 2016;5:15978.

34. Niu C, Li H, Jiang L, et al. Genome-wide identification of drought-responsive microRNAs in two sets of

Cite this article as: Huang $\mathrm{P}$, Wei S, Luo M, Tang Z, Lin Q, Wang X, Luo M, He Y, Wang C, Wei D, Xia C, $\mathrm{Xu}$ J. MiR-139-5p has an antidepressant-like effect by targeting phosphodiesterase 4D to activate the cAMP/PKA/CREB signaling pathway. Ann Transl Med 2021;9(20):1594. doi: 10.21037/atm-21-5149
Huang et al. MiR-139-5p is antidepressant by targeting PDE4D.

Malus from interspecific hybrid progenies. Hortic Res 2019;6:75.

(English Language Editor: A. Muijlwijk) 


\section{Supplementary}

A

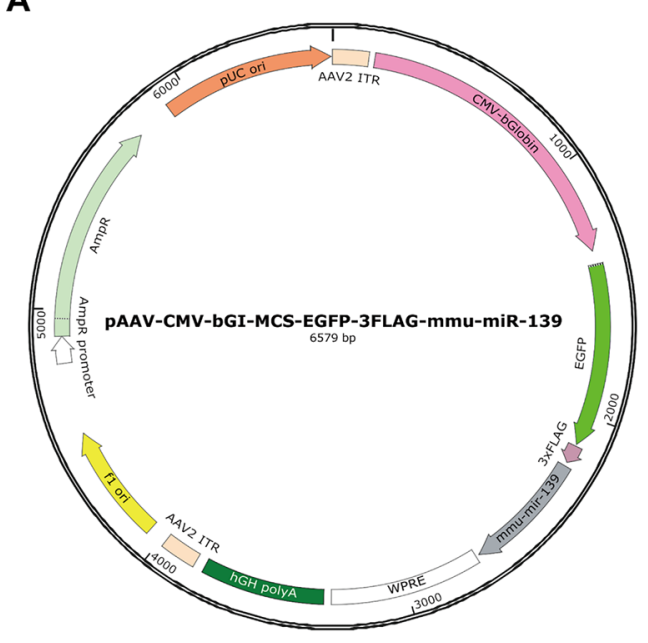

C

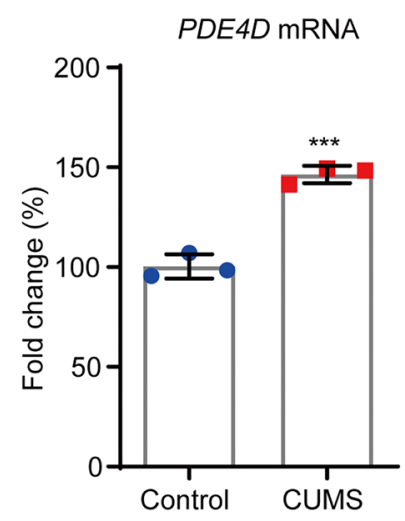

B

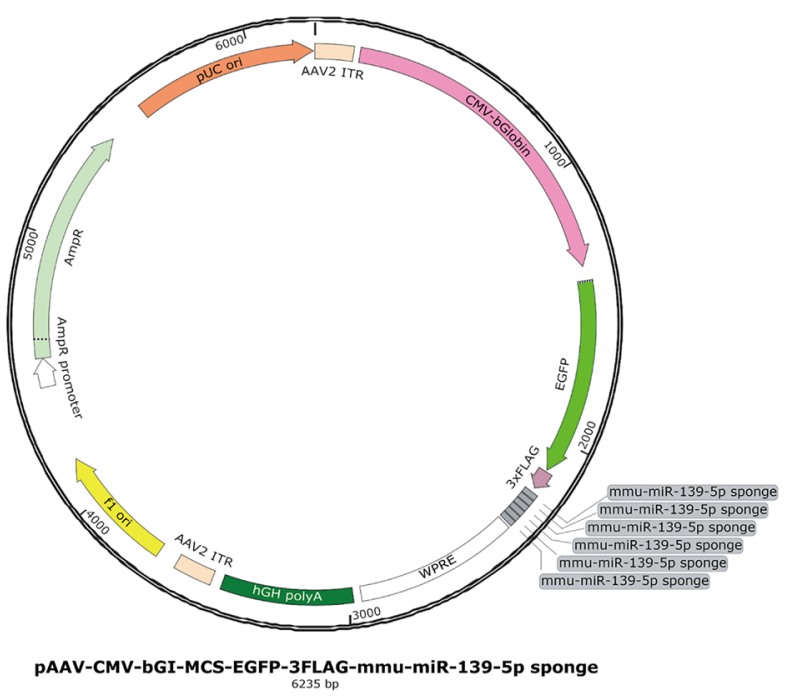

D
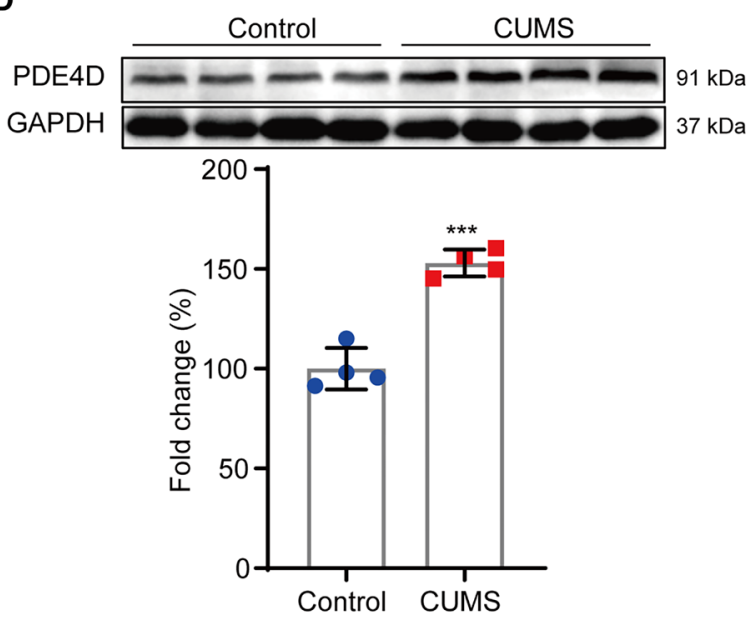

Figure S1 Expression level of PDE4D in hippocampus region of mouse brain, and schematic illustration of design and construction of recombinant AAV vectors. (A, B) A schematic illustration of design and construction of recombinant AAV-miR-139-5p and AAV-miR-1395p sponge. Relative PDE4D mRNA (C) and protein (D) expression were quantified by qPCR and western blotting, respectively. ${ }^{* * *} \mathrm{P} \leq 0.01$ versus the control group. All data are presented as mean \pm SEM. PDE4D, phosphodiesterase 4D; AAV, adeno-associated virus. 
Table S1 Sequences of the oligo primers used in this study

\begin{tabular}{|c|c|c|}
\hline Primers & \multicolumn{2}{|c|}{ Primer sequence } \\
\hline miR-139-5p & АCACTCCAGCTGGGTCTACAGTGCACGTGTC & ACTGACTGATGCAATCTCAACTGGTGTCGTGGA \\
\hline miR-335-5p & UGUUUUGAGCGGGGGUCAAGAGC & CUCUCAUUUGCUAUAUUCA \\
\hline miR-340-5p & CCGTTAGTTACGATTCGAAG & AGGCCGCGCGTAGTGATGCAACA \\
\hline miR-103a-3p & ACACTCCAGCTGGGAGCAGCATTGTACAGGG & TGGTGTCGTGGAGTCG \\
\hline miR-19b-3p & TCCGAAGTCAAAACGTACCTA & GTGCAGGGTCCGAGGT \\
\hline miR-18a-5p & TAAGGTGCATCTAGTGCAGAT & GAACATGTCTGCGTATCTC \\
\hline miR-16-5p & TAGCAGCACGTAAATATTGG & GAACATGTCTGCGTATCTC \\
\hline GAPDH & AAGGGCTCATGACCACAGTC & GGATGCAGGGATGATGTTCT \\
\hline
\end{tabular}

PDE4D, phosphodiesterase 4D; BDNF, brain-derived neurotrophic factor.

Table S2 List of antibodies used for western blot and immunofluorescence

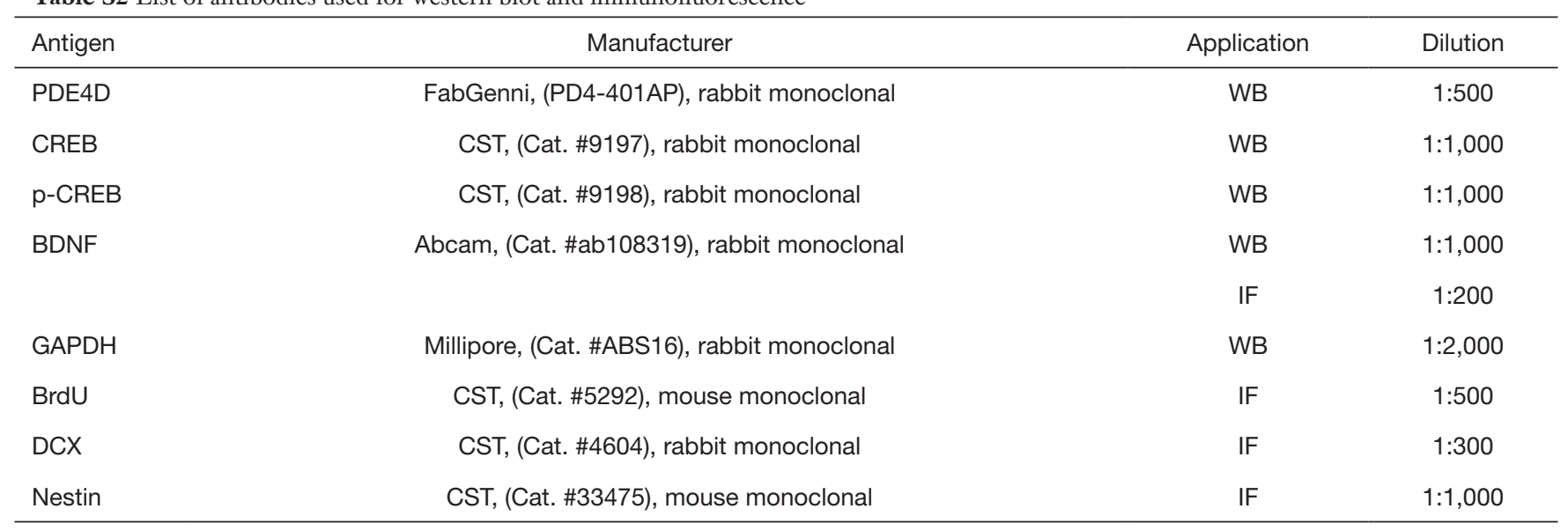

PDE4D, phosphodiesterase 4D; p-CREB, phosphorylated cyclic-AMP response element-binding protein; BDNF, brain-derived neurotrophic factor; BrdU, bromodeoxyuridine; DCX, doublecortin. 